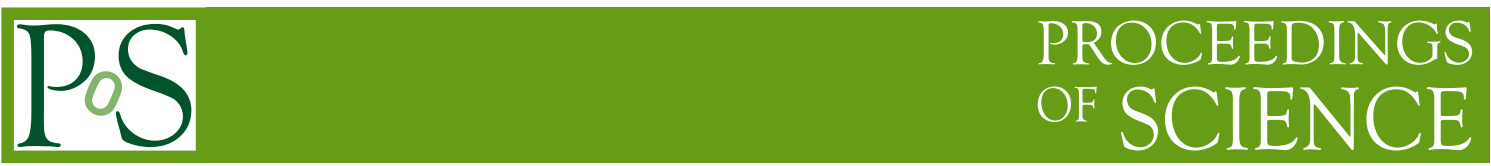

\title{
Differential cross sections for top pair production at the LHC
}

\author{
Marco Guzzi ${ }^{* a}{ }^{\text {Katerina Lipka }}{ }^{a}$ and Sven-Olaf Moch ${ }^{b, c}$ \\ ${ }^{a}$ Deutsches Elektronen-Synchrotron DESY \\ Notkestrasse 85, 22607 Hamburg, Germany \\ ${ }^{b}$ Institut für Theoretische Physik, Universität Hamburg, Luruper Chaussee 149 Hamburg, \\ D-22761, Germany \\ ${ }^{c}$ Deutsches Elektronen-Synchrotron DESY \\ Platanenallee 6, D-15738 Zeuthen, Germany \\ E-mail: marco.guzzi@desy.de,katerina.lipka@desy.de, \\ sven-olaf.mochedesy.de
}

\begin{abstract}
We present results of phenomenological studies for top-quark pair production at the LHC at the center of mass energy $\sqrt{S}=7 \mathrm{TeV}$. The transverse momentum and rapidity distributions for finalstate top quarks are calculated in perturbative QCD at approximate next-to-next-to-leading order $\mathscr{O}\left(\alpha_{s}^{4}\right)$ by using methods of threshold resummation beyond the leading logarithmic accuracy. The theoretical predictions are obtained by using the computer code DIFFToP and are compared to recent measurements by the ATLAS and CMS collaborations. DIFFTOP can be employed in the general case of heavy-quark pair production at hadron-hadron colliders and provides a basis for applications in QCD analyses for parton distribution functions determination.
\end{abstract}

XXII International Workshop on Deep-Inelastic Scattering and Related Subjects 28 April - 2 May 2014, Warsaw, Poland

Preprint DESY $\mathrm{xx}-\mathrm{xxx}$

\footnotetext{
${ }^{*}$ Speaker.
} 
Introduction. Since its discovery at the Tevatron, the top quark has been playing an extremely important role in particle phenomenology.

Its mass, a fundamental parameter of the Standard Model (SM), is the largest in the quark families and it is close to the electroweak symmetry breaking (EWSB) scale. Therefore, the top quark behaves differently with respect to the other quarks for many reasons. The leading decay channel of the top-quark into a $b$ quark and a $W$ boson, is mainly controlled by weak interactions, thus decay properties like spin correlations and helicity can be investigated in a clean way in the decay products, before that hadronization takes place. This is crucial for precision measurements and tests of the electroweak (EW) sector. Furthermore, the mass of the top quark recently obtained from the combined results of the measurements of the CMS and ATLAS [1] collaborations at the LHC, is $m_{t}=173.3 \pm 0.76 \mathrm{GeV}$ and since it is close to the mass of the Higgs boson, it gives us the possibility of studying the interplay between the Higgs sector and top-quark physics.

Due to the large mass, processes involving final-state top quarks in high-energy hadronic reactions are excellent candidates to probe parton distribution functions (PDFs) of the proton in kinematic regions where these (particularly the gluon) are currently poorly constrained and are correlated with the strong coupling constant $\alpha_{s}$ and top-quark mass $m_{t}$.

LHC run-I provided us with the possibility to perform precise measurements of total and differential cross sections for top-quark pair production at center-of-mass energies $\sqrt{S}=7$ and $8 \mathrm{TeV}$, recently published by the CMS $[2,3]$ and ATLAS $[4,5,6]$ collaborations. These measurements are being used in multiple phenomenological analyses where $t \bar{t}$ data are used to test the properties of the Standard Model (SM), QCD factorization and to investigate possible signals of physics beyond the SM (BSM).

Total and differential cross sections for $t \bar{t}$ pair production at the LHC are mostly driven by the gluon-gluon luminosity, in which the gluon PDF is probed at large values of the parton momentum fraction $x \approx 0.1$. The inclusion of $t \bar{t}$ pair production measurements in global QCD analyses for PDF determinations will allows us to investigate the correlation between the top-quark mass $m_{t}$, strong coupling constant $\alpha_{s}$, and the gluon.

In this brief paper we illustrate phenomenological results, documented in Refs. [7, 8], which are of importance for analyses at the LHC and are obtained by using the DIFFToP code, which provides a basis for applications in QCD analyses to determine PDFs and it will be soon released for public use.

The need for precision. To fully exploit the constraining power of the current data, precise theoretical predictions are needed at the highest perturbative order possible, in which systematic uncertainties associated with renormalization/factorization $\left(\mu_{R}, \mu_{F}\right)$ and other scales are reduced. Efficient tools for the analyses, incorporating the current state-of-the-art of QCD calculations for $t \bar{t}$ observables are therefore necessary. The QCD corrections to heavy-quark production at hadron colliders at the next-to-leading order (NLO), $\mathscr{O}\left(\alpha_{s}^{3}\right)$, are known since many years $[9,10,11,12,13,14]$. The full calculation at next-to-next-to-leading order (NNLO), $\mathscr{O}\left(\alpha_{s}^{4}\right)$, for the inclusive cross section has been accomplished only recently $[15,16,17,18]$ and required continuous efforts of the QCD community in calculating radiative corrections and in the development of computational tools $[19,20,21,22,23,24,25,26]$. The NNLO calculation for the inclusive cross section for the $t \bar{t}$ production is implemented in the C++ computer programs ToP++ [27] and HATHOR [28]. The exact NLO calculations for $t \bar{t}$ total and differential cross sections are ef- 
ficiently implemented into Monte Carlo (MC) numerical codes MCFM [29], MC@NLO [30], aMCfast [31], POWheg [32], MadGraph/MadEvent [33, 34]. On the other hand, the NNLO corrections for $t \bar{t}$ differential cross sections are not yet available and NLO predictions seem to be not accurate enough to describe the current LHC data, because perturbative corrections are large and systematic uncertainties associated to various scales entering the calculation are important (see Fig. 1). For this specific purpose, techniques of QCD threshold resummation provide us with theoretical tools to estimate the importance of perturbative higher orders in cross-section calculations [35, 36, 37, 38, 39, 40]. By using threshold resummation methods one can derive approximate formulas beyond the NLO approximation, in which cross sections are expanded in terms of the logarithmic enhanced contributions and can therefore be written at various degrees of logarithmic accuracy.

DIFFTOP is a Mellin-space resummation computer code for calculating total and differential cross section for heavy-flavor production at hadron colliders at approximate NNLO $\mathscr{O}\left(\alpha_{s}^{4}\right)$. It uses techniques of logarithmic expansion beyond the leading logarithmic accuracy in QCD threshold resummation and the implementation strictly follows the derivation in Ref. [41] and references therein. Other particulars of the calculation can be found in Refs. [7, 42, 43, 44, 45].

For the purpose of a fast calculation within QCD analyses for PDF determination, DIFFToP has been interfaced to FASTNLO [46, 47, 48] which allows the user to calculate fast theory predictions using any PDFs. DIFFTOP and its interface to FASTNLO provide a framework for the inclusion of differential $t \bar{t}$ cross sections at approximate NNLO into QCD analyses of PDFs, where a simultaneous determination of gluon, $\alpha_{s}\left(M_{Z}\right)$ and top-quark mass, using the $t \bar{t}$ measurements together with measurements of Deep-Inelastic Scattering (DIS) and jet production in DIS and protonproton collisions, might resolve the correlations among these quantities.

Results. In this section we show results for the approximate NNLO calculation for differential cross sections in the single-particle inclusive (1PI) kinematic at the LHC (see Refs.[39, 41] for details on the kinematics). Theory predictions are compared to the recent LHC measurements of differential distributions for $t \bar{t}$ production at $\sqrt{S}=7 \mathrm{TeV}$ by the CMS [2] and ATLAS [5] collaborations. In particular, transverse momentum $p_{T}^{t}$ distributions for the final-state top quark are presented here. The central prediction corresponding to the approximate NNLO is obtained by choosing $m_{t}=173$ (pole) $\mathrm{GeV}$, and renormalization and factorization scales as $\mu_{R}=\mu_{F}=m_{t}$. The theory predictions shown for the various PDF sets ABM11 [49], CT10 [50], HERAPDF1.5 [51], MSTW08 [52], and NNPDF2.3 [53], use the $\alpha_{s}\left(M_{Z}\right)$ value given by each group. In Fig. 1 we compare DIFFTOP and the full NLO calculation obtained by MCFM [29]. The inclusion of higher orders reduces substantially the scale dependence and also modifies the shape of the $p_{T}^{t}$ distribution. When these theory predictions are compared to the recent LHC measurements the theoretical description of the measurements significantly improves in the NNLO case. In Fig. 2 we compare predictions using different PDF sets to the CMS and ATLAS data. The errors corresponding to the different PDF sets are represented by bands with different hatches and are estimated by summing in quadrature the uncertainties relative to PDF, $\alpha_{s}\left(M_{Z}\right)$, scale, and $m_{t}$ variations. Here PDF uncertainties are shown at $68 \%$ confidence level (CL) and are computed by following the prescription given by each PDF group with the exception of ABM, in which the total uncertainty, obtained with the symmetric formula for the eigenvector sets, represents the PDF $+\alpha_{s}$ uncertainty at the $68 \%$ CL. For the MSTW08, CT10, HERAPDF1.5, and NNPDF2.3 PDF sets the uncertainty associated 

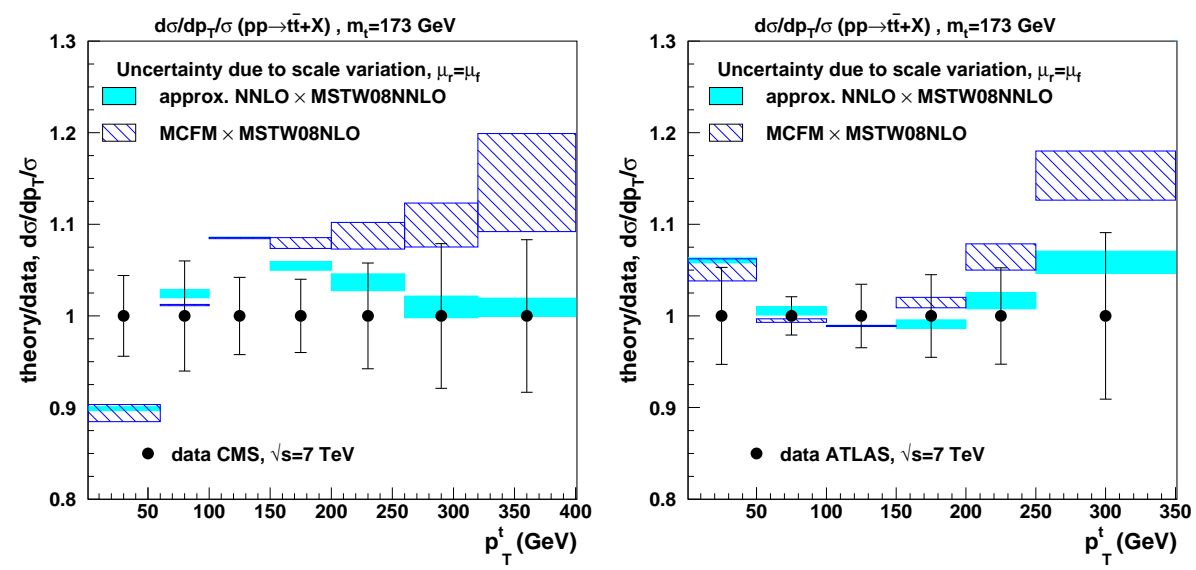

Figure 1: Study of scale uncertainties for MCFM and DifFToP calculations for the top-quark $p_{T}^{t}$ distribution. Ratio of theory over data for CMS (left) [2] and ATLAS (right) [4] measurements. Here MSTW08 NLO (NNLO) PDFs are used for the MCFM (DIFFTOP) calculation. Renormalization and factorization scales are set to $\mu_{R}=\mu_{F}=m_{t}$ and varied such as $m_{t} / 2 \leq \mu_{R}=\mu_{F} \leq 2 m_{t}$.

to $\alpha_{s}\left(M_{Z}\right)$ is given by the central value as given by each group $\pm \Delta \alpha_{s}\left(M_{Z}\right)=0.001$. The scale uncertainty is obtained by variations $m_{t} / 2 \leq \mu_{R}=\mu_{F} \leq 2 m_{t}$, while the uncertainty associated to the top-quark mass is estimated by using $m_{t}=173 \mathrm{GeV}$ (pole mass) $\pm \Delta m_{t}=1 \mathrm{GeV}$.

At the present stage, even though the CMS and ATLAS measurements exhibit relatively large uncertainties, these data might have some impact in PDFs determination once included in QCD fit analyses. On the other hand, a significant amount of information contained in the measurements of differential distributions is lost by normalizing the data. Measurements of absolute differential cross sections are of crucial importance to fully exploit the potential of the $t \bar{t}$ production to constrain the gluon distribution. Moreover, a reduction of the statistic and systematic uncertainties in the high-energy run of the LHC will be of clear advantage.

Conclusions. We have shown results for $t \bar{t}$ differential cross sections obtained with the flexible computer code DIFFTOP at approximate NNLO, which are relevant for phenomenological investigations at the hadron colliders. We have illustrated theoretical predictions for PDF sets that account for uncertainties due to variations of PDFs, scale, $\alpha_{s}$ and $m_{t}$, and that are compared to the recent measurements by the CMS and ATLAS collaborations. Given the accuracy of the present data and the existing correlations between the strong coupling $\alpha_{s}\left(M_{Z}\right)$, top-quark mass, and gluon PDF, these measurements might have an impact in constraining the large- $x$ gluon distribution once included in QCD fit of PDFs. In particular, investigations of absolute differential cross sections will bring complementary information related to the magnitude and other details of the distributions, which will be crucial to improve the constraining power of the experimental data.

Acknowledgments. This work was supported by the "Initiative and Networking Fund of the Helmholtz Association (HGF) under the contract S0-072".

\section{References}

[1] ATLAS, CDF, CMS, and D0 Collaborations, (2014), 1403.4427. 

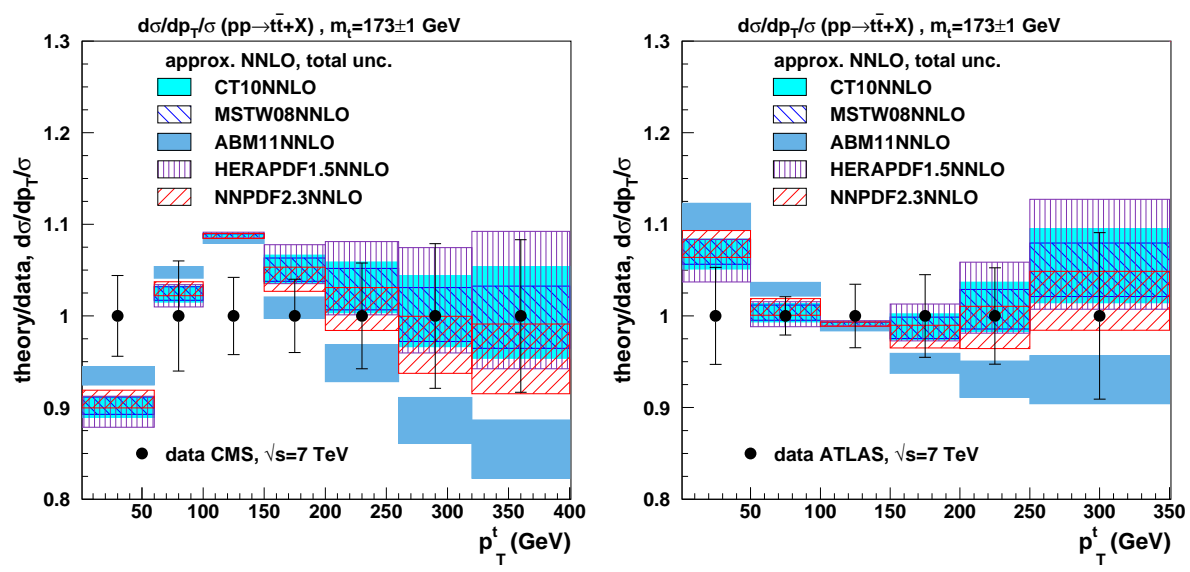

Figure 2: The approx. NNLO predictions for top-quark pair production at the LHC at $\sqrt{S}=7 \mathrm{TeV}$, shown as functions of $p_{T}^{t}$. The predictions, obtained by using different PDF sets are presented as a ratio to the LHC measurements (filled symbols). Predictions, obtained by using different PDF sets are presented by bands of different hatches. The total uncertainty is obtained by summing the uncertainties due to PDFs, $\alpha_{s}, m_{t}$ and scale variations in quadrature.

[2] CMS Collaboration, S. Chatrchyan et al., Eur.Phys.J. C73 (2013) 2339, 1211.2220.

[3] CMS Collaboration, S. Chatrchyan et al., (2013), 1312.7582.

[4] ATLAS Collaboration, G. Aad et al., Eur.Phys.J. C73 (2013) 2261, 1207.5644.

[5] ATLAS collaboration, G. Aad et al., ATLAS-CONF-2013-099, ATLAS-COM-CONF-2013-114 (2013).

[6] ATLAS collaboration, G. Aad et al., ATLAS-CONF-2013-097, ATLAS-COM-CONF-2013-112 (2013).

[7] M. Guzzi, K. Lipka and S.O. Moch, (2014), 1406.0386.

[8] M. Guzzi, K. Lipka and S.O. Moch, PoS DIS2013 (2013) 049, 1308.1635.

[9] P. Nason, S. Dawson and R.K. Ellis, Nucl.Phys. B303 (1988) 607.

[10] P. Nason, S. Dawson and R.K. Ellis, Nucl.Phys. B327 (1989) 49.

[11] W. Beenakker et al., Phys.Rev. D40 (1989) 54.

[12] R. Meng et al., Nucl.Phys. B339 (1990) 325.

[13] W. Beenakker et al., Nucl.Phys. B351 (1991) 507.

[14] M.L. Mangano, P. Nason and G. Ridolfi, Nucl.Phys. B373 (1992) 295.

[15] M. Czakon, P. Fiedler and A. Mitov, Phys.Rev.Lett. 110 (2013) 252004, 1303.6254.

[16] M. Czakon and A. Mitov, JHEP 1301 (2013) 080, 1210.6832.

[17] M. Czakon and A. Mitov, JHEP 1212 (2012) 054, 1207.0236.

[18] P. Bärnreuther, M. Czakon and A. Mitov, Phys.Rev.Lett. 109 (2012) 132001, 1204.5201. 
[19] M. Czakon, A. Mitov and S. Moch, Nucl.Phys. B798 (2008) 210, 0707.4139.

[20] M. Czakon, A. Mitov and S. Moch, Phys.Lett. B651 (2007) 147, 0705.1975.

[21] A. Mitov and S. Moch, JHEP 0705 (2007) 001, hep-ph/0612149.

[22] A. Ferroglia et al., Phys.Rev.Lett. 103 (2009) 201601, 0907.4791.

[23] A. Ferroglia et al., JHEP 0911 (2009) 062, 0908.3676.

[24] M. Czakon, Phys.Lett. B693 (2010) 259, 1005.0274.

[25] I. Bierenbaum, M. Czakon and A. Mitov, Nucl.Phys. B856 (2012) 228, 1107.4384.

[26] P. Bärnreuther, M. Czakon and P. Fiedler, JHEP 1402 (2014) 078, 1312.6279.

[27] M. Czakon and A. Mitov, (2011), 1112.5675.

[28] M. Aliev et al., Comput.Phys.Commun. 182 (2011) 1034, 1007.1327.

[29] J.M. Campbell and R.K. Ellis, Phys.Rev. D62 (2000) 114012, hep-ph/0006304.

[30] S. Frixione, P. Nason and B.R. Webber, JHEP 0308 (2003) 007, hep-ph/0305252.

[31] V. Bertone et al., (2014), 1406.7693.

[32] S. Alioli et al., JHEP 1104 (2011) 081, 1012.3380.

[33] J. Alwall et al., JHEP 0709 (2007) 028, 0706.2334.

[34] R. Frederix et al., JHEP 0910 (2009) 003, 0908.4272.

[35] G.F. Sterman, Nucl.Phys. B281 (1987) 310.

[36] S. Catani and L. Trentadue, Nucl.Phys. B327 (1989) 323.

[37] S. Catani and L. Trentadue, Nucl.Phys. B353 (1991) 183.

[38] N. Kidonakis and G.F. Sterman, Nucl.Phys. B505 (1997) 321, hep-ph/9705234.

[39] E. Laenen, G. Oderda and G.F. Sterman, Phys.Lett. B438 (1998) 173, hep-ph/9806467.

[40] R. Bonciani et al., Nucl.Phys. B529 (1998) 424, hep-ph/9801375.

[41] N. Kidonakis et al., Phys.Rev. D64 (2001) 114001, hep-ph/0105041.

[42] N. Kidonakis, Phys.Rev. D64 (2001) 014009, hep-ph/0010002.

[43] N. Kidonakis, Int.J.Mod.Phys. A19 (2004) 1793, hep-ph/0303186.

[44] N. Kidonakis, Phys.Rev. D73 (2006) 034001, hep-ph/0509079.

[45] N. Kidonakis, (2013), 1311.0283.

[46] fastNLO Collaboration, D. Britzger et al., (2012) 217, 1208.3641.

[47] fastNLO Collaboration, M. Wobisch et al., (2011), 1109.1310.

[48] T. Kluge, K. Rabbertz and M. Wobisch, (2006) 483, hep-ph/0609285.

[49] S. Alekhin, J. Blümlein and S. Moch, Phys.Rev. D86 (2012) 054009, 1202.2281.

[50] J. Gao et al., Phys.Rev. D89 (2014) 033009, 1302.6246.

[51] ZEUS Collaboration, H1 Collaboration, A. Cooper-Sarkar, PoS EPS-HEP2011 (2011) 320, 1112.2107.

[52] A. Martin et al., Eur.Phys.J. C63 (2009) 189, 0901.0002.

[53] R.D. Ball et al., Nucl.Phys. B867 (2013) 244, 1207.1303. 